\title{
Facoemulsificação e utilização de lentes intra-oculares em portadores de hanseníase: estudo caso-controle
}

\author{
Phacoemulsification with intraocularlens implantation in leprosy patients: \\ case control study
}

\author{
Rodrigo José Nunes Dias ${ }^{1}$ \\ Mônica Jeha Maakaroun ${ }^{2}$ \\ Aldemar Vilela de Castro ${ }^{3}$
}

Hospital Governador Israel Pinheiro do Instituto da Previdência dos Servidores do Estado de Minas Gerais - IPSEMG - Belo Horizonte (MG) - Brasil.

${ }^{1}$ Residente em Oftalmologia do Hospital Governador Israel Pinheiro do Instituto da Previdência dos Servidores do Estado de Minas Gerais - IPSEMG - Belo Horizonte (MG) - Brasil.

${ }^{2}$ Doutora em Oftalmologia pela Universidade Federal de Minas Gerais - UFMG; Oftalmologista do Departamento de Hanseníase do Hospital São Geraldo - UFMG; Oftalmologista da Colônia Santa Isabel da Fundação Hospitalar do Estado de Minas Gerais - FHEMIG Belo Horizonte (MG) - Brasil.

${ }^{3}$ Doutor em Oftalmologia pela Universidade de São Paulo - USP - São Paulo (SP) - Brasil; Coordenador da Clínica Oftalmológica do Hospital Governador Israel Pinheiro do IPSEMG - Belo Horizonte (MG) - Brasil.

Endereço para correspondência: Rodrigo José Nunes Dias. Rua Paracatu, 860 - Apto. 201 - Belo Horizonte (MG) CEP 30180-090

E-mail: rnunesdias@hotmail.com

Recebido para publicação em 30.05.2005

Versão revisada recebida em 15.11.2005

Aprovação em 29.11.2005

\section{RESUMO}

Objetivo: Verificar as principais alterações e complicações relacionadas à cirurgia de catarata com o implante de lente intra-ocular em portadores de hanseníase, utilizando-se a técnica da facoemulsificação e, comparar estes resultados com um grupo de pacientes sem a doença. Métodos: O método utilizado foi o estudo caso-controle, no qual foram incluídos 31 olhos de hansenianos operados no período de junho de 1999 a janeiro de 2003, denominados de Grupo Caso. Paralelamente foram selecionados outros 31 olhos de pacientes não hansenianos denominados de Grupo Controle. As comparações entre os Grupos foram realizadas pelo teste exato de Fisher. Foi utilizado programa de processamento estatístico EPI INFO versão 6.04. Resultados: Na análise das complicações pós-operatórias, apenas a rotura de esfíncter evidenciou relação com a hanseníase, com um p=0,024. A distribuição da frequiência das demais alterações não mostrou significância estatística ( $p>0,05)$. A melhora na acuidade visual foi semelhante nos dois grupos. Conclusão: O estudo comprovou que, quanto à acuidade visual no pós-operatório e as complicações, os pacientes hansenianos assemelham-se à população em geral, quando utilizada a técnica da facoemulsificação com introdução de lente intra-ocular.

Descritores: Catarata; Hanseníase; Facoemulsificação; Lentes intra-oculares; Período pós-operatório; Estudo de casos e controles

\section{INTRODUÇÃO}

A hanseníase freqüentemente afeta o olho e atualmente é uma importante causa de cegueira em países onde é endêmica. Essa doença ocorre principalmente em regiões subdesenvolvidas do globo, sobretudo na África, Sudeste Asiático e América do Sul. Apresenta uma incidência de 7,34 por 10.000 habitantes e a prevalência de 14,05 por 10.000 habitantes $^{(1)}$.

Dentre as alterações oculares relacionadas com a hanseníase, a catarata se constitui na principal causa de baixa acuidade visual nos portadores e sua prevalência tem aumentado em virtude da longevidade da população associada à insuficiência de serviços especializados. Nos hansenianos a catarata pode ser senil, secundária à terapia crônica com esteróides ou ainda estar associada com uveíte crônica ${ }^{(2)}$.

Courtright et al. em 1998 estimou entre 350 a 400 mil hansenianos com cegueira no mundo, dentre estes cerca de metade dos casos se associava à catarata $^{(3)}$. Em outro estudo, multicêntrico, foram avaliados 849 olhos cegos em hansenianos, metade dos quais estavam direta ou indiretamente relacionados com catarata ${ }^{(4)}$. No Brasil, a prevalência de catarata em portadores de hanseníase variou de $24,6 \%$ até $58,7 \%{ }^{(1,5)}$. 
A cegueira decorrente da catarata é evitável, sendo a cirurgia o único tratamento. As técnicas mais utilizadas são a facectomia extra-capsular (FEC) e a facoemulsificação (FACO), ambas com o implante de lente intra-ocular (LIO) em substituição ao cristalino.

A utilização da LIO é patente. A simplicidade das técnicas atuais com excelente qualidade de visão final aliada ao fato de alguns destes pacientes apresentarem graves sequielas incapacitantes sobrepõe a antigos conceitos e levam a uma melhor qualidade de vida a estes pacientes.

Na literatura mundial há carência de estudos sobre a utilização de LIO em portadores de hanseníase. Nos poucos trabalhos apresentados não se verifica a comparação deste grupo de pacientes com um grupo de pacientes sadios, isentos da patologia. Pouco se descreve sobre os resultados da acuidade visual e das complicações pós-operatórias. Este estudo pretende verificar as principais alterações e complicações pré, per e pós-operatórias de catarata com implantação de lente intraocular em hansenianos através da facoemulsificação e comparar estes resultados com um grupo de pacientes sem a doença.

\section{MÉTODOS}

Nesse estudo foram incluídos 31 olhos de portadores de hanseníase submetidos à cirurgia de catarata através da técnica de facoemulsificação com implantação de LIO no período de junho de 1999 a janeiro de 2003, operados no Hospital São Geraldo (UFMG) e Hospital Governador Israel Pinheiro (IPSEMG) e acompanhados pelo departamento de oftalmologia da Colônia Santa Isabel (FHEMIG). Estes pacientes foram denominados de Grupo Caso.

Paralelamente, foram também selecionados outros 31 olhos de pacientes não hansenianos, por amostragem pareada, idade e mesma técnica cirúrgica. Estes foram denominados de Grupo Controle. A análise dos grupos foi na proporção de 1:1, sendo operados no mesmo período de tempo.

No pré-operatório, todos os pacientes foram submetidos à avaliação clínica e oftalmológica. $\mathrm{O}$ exame oftalmológico incluiu a medida da acuidade visual com a tabela de Snellen, biomicroscopia, medida da pressão ocular (PO), oftalmoscopia, ceratometria, ecobiometria e cálculo da LIO. A ecografia foi indicada quando a oftalmoscopia era inviável. A acuidade visual era sempre igual ou inferior a 20/200 no Grupo Caso e a PO menor que $20 \mathrm{mmHg}$.

No Grupo Caso foi realizado ainda uma avaliação dermatológica para revisão das lesões relacionadas com a hanseníase, e para verificar o estágio de tratamento e história de reação recente, sendo que os pacientes eram liberados para cirurgia após estarem livres de reações por um período de seis meses.

De acordo com o tipo de catarata, no Grupo Caso elas foram subdivididas em senil, associada à atrofia de íris e complicada, esta última provavelmente secundária a reação inflamatória crônica com presença de sinéquia posterior. O Grupo Controle apresentava somente o tipo senil.
As pupilas foram dilatadas no pré-operatório com colírio de tropicamida a $1 \%$ e fenilefrina $10 \%$. O bloqueio anestésico utilizado foi o peribulbar com associação de marcaína a 0,75\% e lidocaína a 2\%. Foi realizada: paracentese na câmara anterior, capsulorrexe circular contínua com cistítomo, hidrodissecção e hidrodelineação. Quanto à emulsificação do núcleo, esta variou de acordo com sua consistência, sendo realizada a técnica dividir para conquistar na maioria dos casos. Posteriormente, o córtex restante foi aspirado, material viscoelástico (metilcelulose $2 \%$ ) foi injetado dentro do saco capsular e a LIO inserida, sendo esta de 5,25 mm peça única de polimetilmetacrilato. Foi realizada injeção subconjuntival de $0,5 \mathrm{ml}$ de dexametasona. No pós-operatório, foram instituídas corticoterapia e antibioticoterapia tópicas. O pré, per e pós-operatório foram similares em todos os casos.

No pós-operatório, os pacientes foram examinados no $1^{\circ} \mathrm{e}$ $3^{\circ}$ dia, na semana seguinte, 30 e 60 dias. Alguns pacientes foram seguidos por um período de 6 meses. Os dados utilizados foram os obtidos do $2^{\circ}$ até o $6^{\circ}$ mês de pós-operatório. Neste período foram avaliados a acuidade visual, as complicações que foram identificadas pela biomicroscopia e o grau de satisfação dos pacientes em relação à cirurgia através de questionário próprio.

A análise da pesquisa foi realizada através do programa de processamento estatístico EPI INFO versão 6.04. Foram feitas as distribuições das freqüências das variáveis e calculadas as taxas de prevalência de modo usual.

As comparações entre os Grupos Caso e Controle foram realizadas utilizando-se o Teste Exato de Fisher. Os níveis de significância estatística foram de $5 \%$ e dos intervalos de confiança de $95 \%$.

\section{RESULTADOS}

Foram estudados 62 olhos, sendo 31 de portadores de hanseníase utilizados no Grupo Caso e 31 de não portadores correspondendo ao Grupo Controle.

Destes 62 olhos, cerca de $28(45,16 \%)$ eram de pacientes do sexo feminino e $34(54,8 \%)$ eram de pacientes do sexo masculino.

Quanto à idade, esta variou de 42 a 88 anos, com uma média de 66,8 anos e desvio padrão de 12,1 anos.

Em relação ao tipo de catarata, o Grupo Caso apresentou 20 olhos $(64,51 \%)$ com o tipo senil, 8 olhos $(25,8 \%)$ associada à atrofia de íris e 3 olhos $(9,67 \%)$ com catarata complicada. No Grupo Controle todos os pacientes apresentavam apenas catarata senil (Tabela 1).

\begin{tabular}{|lrrrrr|}
\hline \multicolumn{3}{|l}{ Tabela 1. Caracterização dos pacientes segundo o tipo de catarata } \\
\cline { 2 - 3 } Tipo de catarata & Hanseníase & & \multicolumn{2}{c|}{ Normal } \\
\cline { 2 - 3 } \cline { 5 - 6 } Senil & $\mathbf{N}$ & $\%$ & & $\mathbf{N}$ & $\%$ \\
Atrofia de íris & 8 & 64,51 & & 31 & 100 \\
Complicada & 3 & 9,67 & & 0 & 0 \\
Total & 31 & 100,00 & & 31 & 100 \\
\hline
\end{tabular}




\begin{tabular}{|c|c|c|c|c|c|}
\hline \multirow{2}{*}{ Complicações } & \multicolumn{2}{|c|}{ Grupo caso } & \multicolumn{2}{|c|}{ Grupo controle } & \multirow{2}{*}{$\mathbf{p}$} \\
\hline & $\mathbf{N}$ & $\%$ & $\mathbf{N}$ & $\%$ & \\
\hline Captura LIO & 1 & 3,2 & 0 & 0 & 1,000 \\
\hline Sem captura LIO & 30 & 96,8 & 31 & 100 & \\
\hline Com sinéquia & 4 & 12,9 & 1 & 3,2 & 0,354 \\
\hline Sem sinéquia & 27 & 87,1 & 30 & 96,8 & \\
\hline LIO descentrada & 1 & 3,2 & 1 & 3,2 & 1,000 \\
\hline LIO eutópica & 30 & 96,8 & 30 & 96,8 & \\
\hline Com depósitos & 1 & 3,2 & 0 & 0 & 1,000 \\
\hline Sem depósitos & 30 & 96,8 & 31 & 100 & \\
\hline Rotura esfíncter & 6 & 19,4 & 0 & 0 & 0,024 \\
\hline Sem rotura esfíncter & 25 & 80,6 & 31 & 100 & \\
\hline Opacidade capsular & 3 & 9,7 & 0 & 0 & 1,000 \\
\hline Sem opacidade capsular & 28 & 90,3 & 31 & 100 & \\
\hline Com inflamação & 1 & 3,2 & 0 & 0 & 1,000 \\
\hline Sem inflamação & 30 & 96,8 & 31 & 100 & \\
\hline
\end{tabular}

Em relação ao grau de satisfação dos pacientes após a cirurgia, $29(93,6 \%)$ dos pacientes do Grupo Caso e $30(96,8 \%)$ do Grupo Controle estavam satisfeitos com o resultado.

\section{DISCUSSÃO}

A catarata é considerada a principal causa de cegueira em portadores de hanseníase. A incidência aumenta com a idade, podendo estar associada com sinéquia e também com atrofia de íris e miose ${ }^{(2)}$.

Alguns trabalhos observaram que a prevalência de catarata em pacientes com hanseníase vem aumentando com a maior expectativa de vida dos mesmos ${ }^{(6)}$.

Em nosso estudo, o Grupo Caso, no pré-operatório apresentou $64,51 \%$ de catarata senil, $25,8 \%$ de catarata associada à atrofia de íris e 9,67\% de catarata complicada.

Em um estudo descritivo, em 1998, onde se utilizou a técnica da facectomia extra-capsular (FEC) em portadores de hanseníase foi encontrado no pós-operatório as seguintes alterações: captura de LIO, sinéquias, LIO descentrada, depósitos na LIO e sinais de inflamação ${ }^{(7)}$.

Em um estudo nacional em 2001, o autor verificou que a presença de alterações inflamatórias decorrentes da cirurgia (FEC e FACO) com implantação de LIO eram mais freqüentes no grupo de pacientes hansenianos ${ }^{(8)}$.

\begin{tabular}{|c|c|c|c|c|c|}
\hline \multirow[t]{2}{*}{ Acuidade visual } & \multicolumn{2}{|c|}{ Grupo caso } & \multirow{2}{*}{$\frac{\text { Grupo }}{N}$} & \multirow{2}{*}{$\begin{array}{c}\text { controle } \\
\%\end{array}$} & \multirow[t]{2}{*}{$\mathbf{p}$} \\
\hline & $\mathbf{N}$ & $\%$ & & & \\
\hline$\geq 20 / 40$ & 26 & 83,9 & 27 & 87,1 & 1,000 \\
\hline $20 / 60 \leq A V \geq 20 / 100$ & 3 & 9,7 & 3 & 9,7 & \\
\hline$\leq 20 / 200$ & 2 & 6,5 & 1 & 3,2 & \\
\hline Total & 31 & 100,0 & 31 & 100,0 & \\
\hline
\end{tabular}

A rotura de esfíncter, que foi evidenciada pelo aspecto biomicroscópico da íris, com presença de atonia e irregularidade da pupila, neste trabalho foi verificada em $19,4 \%$ dos pacientes do grupo caso, enquanto que o grupo controle não apresentou esta alteração.

$\mathrm{Na}$ análise, a rotura de esfíncter evidenciou relação com a hanseníase com um $\mathrm{p}=0,024$. Talvez esta complicação pósoperatória evidente no grupo caso seja devido aos fatores associados às cataratas dos portadores de hanseníase como atrofia de íris, miose e em alguns casos a presença de sinéquias. Estes fatores dificultam a cirurgia, podendo levar a estiramento e alterações funcionais da pupila.

A distribuição da freqüência das demais alterações pósoperatórias como captura de LIO, LIO descentrada, depósitos na LIO, opacificação de cápsula posterior, sinais de inflamação e sinéquia em número absoluto foi maior no grupo caso,

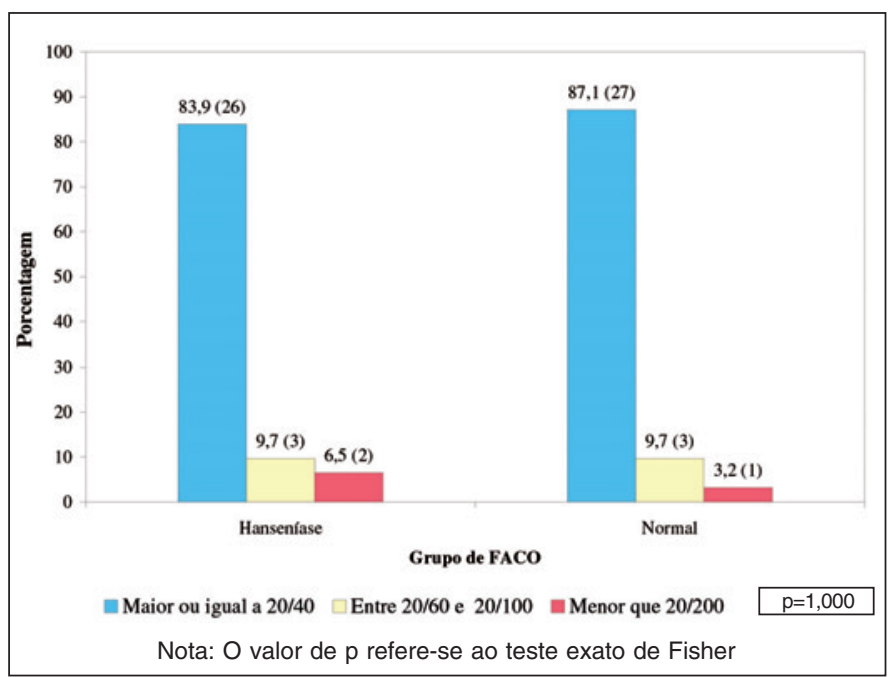

Gráfico 1. Resultados da acuidade visual 
porém quando realizada a análise comparativa, esta não se mostrou estatisticamente significativa com $\mathrm{p}>0,05$. Talvez esta diferença encontrada se deva à técnica cirúrgica utilizada em nosso trabalho, exclusivamente FACO, técnica esta que promove uma menor agressão no ato cirúrgico, com um menor grau de inflamação e complicações pós-operatórias e uma recuperação mais rápida do paciente.

Em 1989, num estudo sobre LIO em hansenianos foi encontrado uma melhora na acuidade visual em $82 \%$ dos pacientes ${ }^{(9)}$.

Em um estudo realizado no Japão em 1996 com a facoemulsificação em portadores de hanseníase, houve uma melhora na acuidade visual em mais de $90 \%$ dos pacientes operados ${ }^{(10)}$.

Ao analisar o pós-operatório em 70 pacientes hansenianos em 1998, a autora verificou um melhor resultado visual em 92,9\% dos pacientes ${ }^{(7)}$.

Em nosso estudo, o Grupo Caso apresentou uma melhor acuidade visual em 29 pacientes $(93,6 \%)$, quando comparamos com o resultado visual do Grupo Controle não encontramos relação estatisticamente significativa $(\mathrm{p}=1,000)$, o que indica um resultado semelhante entre os dois grupos.

No entanto, a acuidade visual AV pós-operatória foi categorizada pelos pesquisadores em AV > 20/40, AV entre 20/60 e 20/100 e AV $<20 / 200$, sendo que a melhora foi considerada como aqueles pacientes que apresentaram AV > 20/100 no grupo caso. Mesmo com esta categorização cerca de 83,9\% dos pacientes hansenianos apresentaram AV > 20/40, o que demonstra que a utilização da LIO associada à técnica cirúrgica de facoemulsificação traz um grande benefício a esta população, melhorando assim sua qualidade de vida e explicando o grau de satisfação apresentado pelos pacientes após a cirurgia, 29 (93,6\%) dos pacientes do Grupo Caso e $30(96,8 \%)$ do Grupo Controle estavam satisfeitos com o resultado.

\section{CONCLUSÃO}

Através deste trabalho podemos concluir:

- Quanto às complicações pós-operatórias da cirurgia de catarata, os pacientes portadores de hanseníase se assemelham à população geral, quando utilizada a técnica da facoemulsificação com introdução de lente intra-ocular.

- O resultado visual pós-operatório é semelhante entre os hansenianos e a população sem a patologia na facoemulsificação com utilização de lente intra-ocular.

\section{ABSTRACT}

Purpose: To check the complications of cataract surgery with intraocular lens implantation in leprosy patients by the phacoemulsification technique, and to compare these results, with a control group. Methods: The used method was a case control study, where 31 eyes from leprosy patients were submited to cataract surgery, from June 1999 to January 2003, called Case Group. On the other hand, 31 eyes of healthy patients were selected for the Control Group. The comparison between the groups was performed using Fisher's exact test. The used program was EPI INFO 6.04. Results: Sphincter tear was the only postoperative complication higher in the leprosy group $(p=0.024)$. The other alterations did not show statistic differences $(\mathrm{p}>0.05)$. Visual acuity improvement was the same in both groups. Conclusions: This study showed that the visual acuity and complications after cataract surgery with intraocular lens are the same in leprosy patients and healthy ones.

Keywords: Cataract; Leprosy; Phacoemulsification; Lenses, intraocular; Postoperative period; Case-control studies

\section{REFERÊNCIAS}

1. Moreno RD, Woods WJ. Prevalência das alterações oculares em pacientes portadores de hanseníase em um Hospital Colônia no Acre. Arq Bras Oftalmol. 1999;62(3):254-7.

2. Hogeweg M. Cataract: the main cause of blindness in leprosy. Lepr Rev. 2001; 72(2):139-42

3. Courtright P. The epidemiology of ocular complications of leprosy. Indian J Lepr. 1998;70(1):33-7. Review.

4. Ffytche TJ. The prevalence of disabling ocular complications of leprosy: a global study. Indian J Lepr. 1998;70(1):49-59.

5. Santos PM, Maradei J, Barra JMM, Santos RCR, Abreu MT. Estudo das alterações oculares em portadores de hanseníase de Hospital Colônia. Arq Bras Oftalmol. 1995;58(2):130-7.

6. Courtright P, Lewallen S, Tungpakorn N, Cho BH, Lim YK, Lee HJ, Kim $\mathrm{SH}$. Cataract in leprosy patients: cataract surgical coverage, barriers to acceptance of surgery, and outcome of surgery in a population based survey in Korea. Br J Ophthalmol. 2001;85(6):643-7.

7. Batistella GG, Maakaroun M, de Castro AV. Extracapsular cataract extraction and intraocular lens implantation in leprosy patients: visual outcome and complications. Indian J Lepr. 1998;70(1):5-10.

8. Almeida EF, Almeida LNF. O uso de lentes intra-oculares em pacientes portadores de hanseníase. Arq Bras Oftalmol. 2001;64(6):541-3.

9. Frota E, Oréfice F. Lente intra-ocular em portadores de Hansen. Rev Bras Oftalmol. 1989;48(4):205-7.

10. Joko S. [Phacoemulsification-aspiration technique with intraocular lens implantation in leprosy patients]. Nihon Hansenbyo Gakkai Zasshi. 1996;65(3):170-3. Janpanese.

Nos artigios enviados para publicação, o nome dos autores e suas afiliações devem estar completos. Isso facilitará a indexação e os links com as bases de dados e o CV Lates. 